J. Bangladesh Acad. Sci., Vol. 42, No. 2, 177-182, 2018

DOI: https://doi.org/10.3329/jbas.v42i2.40048

\title{
EFFECT OF DOPANT CONCENTRATIONS ON MAGNETIC STATES OF Ti, Mn, AND Ni DOPED III-V ZINCBLENDE COMPOUNDS
}

\author{
M. SHAHJAHAN ${ }^{1,2, *}$, T. KHAN ${ }^{1,2}$, M. M. RAHMAN ${ }^{1}$ \\ Department of Physics, University of Dhaka, Dhaka-1000, Bangladesh
}

\begin{abstract}
The effect of Ti, Mn, and Ni dopants concentration on various magnetic states of III-V zincblende compounds $\left(\mathrm{Al}_{1-\mathrm{x}} \mathrm{T}_{\mathrm{x}}\right) \mathrm{M}$ is presented by calculation of multiple scattering theory based on Korringa-Kohn-Rostoker coherent potential approximation (KKR-CPA), where $\mathrm{T}$ denotes $\mathrm{Ti}, \mathrm{Mn}$, and $\mathrm{Ni}$ atoms and $\mathrm{M}$ represents As and $\mathrm{P}$ atoms. The calculations reveal the phase changes in various magnetic states, which are solely controlled by dopant concentrations. The turning points of these behaviors are reported, which are responsible for the change of stable ferromagnetic (FM) state to unstable disordered local moment (DLM) state. The Curie temperature, magnetic moments, and density of states at different concentrations of the dopants are calculated and explain the concurrency with magnetic phase stability.
\end{abstract}

Keywords: KKR-Green’s function, Magnetic States, CPA, Muffin tin potential, Atomic sphere approximation, Curie temperature, Zincblende semiconductors, Density of states.

\section{INTRODUCTION}

Aluminium pnictides have attracted research interest over the few decades due to their unique physical properties such as low densities, high thermal conductivities, wide band gap and large resistivity (Lalngaihawmi et al. 2014; Picket et al. 1989). The binary compounds with the chemical formula AlM belong to the group III-V semiconductors, which crystallizes into zincblende (ZB) structure, where $M$ represents As and $\mathrm{P}$ atoms. The $\mathrm{ZB}$ structure consists of two types of basis atoms compared to the diamond structure having one type of basis atom. The atomic coordinates of the two basis atoms are $(0,0,0)$ and $(1 / 4,1 / 4,1 / 4)$. The solid ZB structure comprises of two inter-penetrating face centered cubic structures shifted by $(1 / 4,1 / 4,1 / 4)$ position along the body diagonal. Introducing transition metals (TM) atom at the cation site, namely the ternary compounds $\left(\mathrm{Al}_{1-\mathrm{x}} \mathrm{T}_{\mathrm{x}}\right) \mathrm{M}$ are of great interest for electronic and magnetic

\footnotetext{
* Corresponding author: <mjahan@du.ac.bd>.

${ }^{1}$ Department of Physics, University of Dhaka, Dhaka-1000, Bangladesh.

${ }^{2}$ Centre for Advanced Research in Sciences, University of Dhaka, Dhaka-1000, Bangladesh.
}

properties with high Curie temperature, where $\mathrm{T}$ indicates $\mathrm{Ti}, \mathrm{Mn}$, and $\mathrm{Ni}$ cations and $\mathrm{x}$ is the cationic (dopant) fractional concentration (Sato et al. 2003).

At present, electronic devices are processed through the integrated chips and magnetic drives to store and manipulate information (Moore 1965). The emerging technological devices will be based on spintronics, which combine both semiconductivity (charge to carry information) and magnetism (spin to store information) to produce a single device. In the field of spintronics, the major tasks are injection, transport and detection of spin polarization of the magnetic atom doped compounds.

Some of the DMS namely (Ga, Mn)As, (Ga, $\mathrm{Mn}) \mathrm{N}$, (Zn, Cr)O, (Zn, Cr)Te, Cu(Al, TM)Se $\mathrm{S}_{2}$ and $\mathrm{Sr}(\mathrm{Ti}, \mathrm{Fe}) \mathrm{O}_{3}$ are considered as the promising spintronic materials (Dietl 2000, Goodenough 1955, Kanamori et al. 2001, Shahjahan et al. 
2016, 2018). The TM atoms have partially filled $d$-states containing unpaired electrons. In terms of their spin moment, they can exhibit ferromagnetic (FM) behavior (Zener 1951). The objective of integration of spin moment was to miniaturize electronic devices to include complex electronic functions in limited space with minimum weight and volume. To perform such functions, several approaches evolved, including micro assembly techniques for individual compounds, then thin film structures and semiconductor integrated circuits.

The realization of unique materials that combine semiconducting behavior with robust magnetism has long been a dream of material scientists. One of the strategies for creating systems that are simultaneously semiconducting and magnetic is to introduce spin moments of electrons into the semiconductors. One of the host cations can be fractionally replaced by TM atoms into semiconducting materials, which are known as dilute magnetic semiconductors (DMS). Recently, the DMS have been extensively studied in order to find ferromagnetism with Curie temperature $\left(\mathrm{T}_{\mathrm{C}}\right)$ higher than room temperature (RT). The DMS being device grade materials have played important role for spintronics application (Wolf et al. 2001). Ferromagnetism is the most attractive materials which can be fabricated by controlled substitution of magnetic atoms at the cationic sites (Anderson et al. 1955). In this article, the effect of $\mathrm{Ti}, \mathrm{Mn}$, and $\mathrm{Ni}$ dopants on various magnetic states and electronic structures of aluminium pnictides have been investigated. The FM state is calculated for the dopants and analyses the magnetic phase stability in terms of the lower energy consideration. In addition to the FM state, the DLM state is calculated, which can be energetically higher or lower than the FM state energy. The Curie temperature is calculated for the FM materials and electronic density of states are plotted to explain the induced magnetic properties.

\section{METHODS OF CALCULATION}

The density functional theory by Kohn-Sham formalism is solved by self-consistent KorringaKohn-Rostoker (KKR) Green’s function method (Kohn et al. 1965). The self-consistency in energy calculation is achieved to a level of 1 micro Rydberg energy level. Generalized gradient approximation (GGA), muffin tin (MT) potential approximation and atomic sphere approximation (ASA) are used in the calculation. The MT potential is considered to be gently touched spheres of spherically symmetric potential, whereas ASA is considered to be slightly overlapping spheres covering the interstitial voids. The Ti, Mn, and Ni dopants are introduced randomly into the cation sites of the host semiconductors, which are well described by the coherent potential approximation (CPA) technique (Shiba 1971). The angular momentum quantum number is specified at each atomic site as $\ell=2$ and $\ell=0$ for $d$ and $s$ electrons, respectively. The solid structures are close packed by considering two more dummy atoms with zero atomic charge, which is simply $s$ electronic state calculation. Irreducible part of the first Brillouin zone of the host crystal is sampled with $256 \mathrm{k}$ sampling points. The lattice constants of the host ZB crystal structures of AlAs and AlP are $5.66 \AA$ and $5.45 \AA$, which are indeed considered for the calculations of doped compounds, respectively (Wyckoff 1963). The numerical computation is performed by the KKR-CPA package "Machikaneyama" developed by Akai (Akai 2002). 


\section{RESULTS AND DISCUSSION}

The FM and DLM calculations of the group III$\mathrm{V}$ type DMS such as $\left(\mathrm{Al}_{1-\mathrm{x}} \mathrm{T}_{\mathrm{x}}\right) \mathrm{As}$, and $\left(\mathrm{Al}_{1-\mathrm{x}} \mathrm{T}_{\mathrm{x}}\right) \mathrm{P}$ have been performed and the following results have been obtained. Stable, unstable, and nonmagnetic states, electronic structures, magnetic moments, and Curie temperature $\left(\mathrm{T}_{\mathrm{C}}\right)$ have been obtained. Surprisingly, at some dilute limit $(0-20 \%)$ of dopants, nonmagnetic states arises with zero local and global magnetic moments. On the other hand, in some cases spin arrangements are such that the DLM state rather than FM state becomes lower in total energy concentration of the three dopants for ASA calculations, as shown in Fig. 1(a). In the case of Ni doping, negative $\Delta \mathrm{E}$ is found, as shown in Fig.1 (b), which implies the higher energy situation of FM state. Since positiveE indicates the FM ground state energy, therefore, Ti in AlAs and Mn in AlP exhibit FM stability, otherwise, the system becomes either nonmagnetic or unstable in FM state. General trend is that the value of $\Delta \mathrm{E}$ is higher for dopants with higher concentrations, which consistently agree with the prediction given by Dietl group (Dietl et al. 2000).
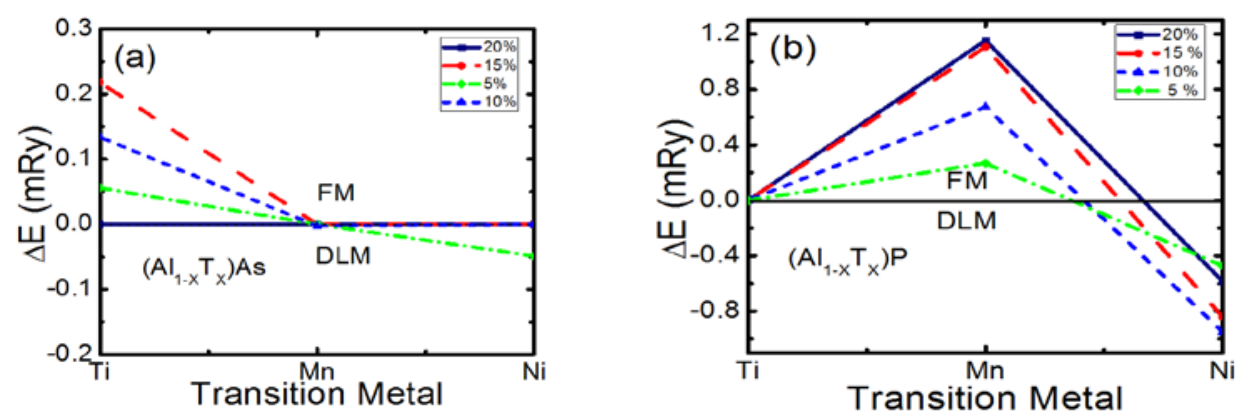

Fig. 1. The energy difference $(\Delta E)$ per unit cell between FM and DLM states for transition metal doped in (a) $\left(\mathrm{Al}_{1-\mathrm{x}} \mathrm{T}_{\mathrm{x}}\right) \mathrm{As}$, and (b) $\left(\mathrm{Al}_{1-\mathrm{x}} \mathrm{T}_{\mathrm{x}}\right) \mathrm{P}$, where $\mathrm{T}$ indicates $\mathrm{Ti}$, $\mathrm{Mn}$ and $\mathrm{Ni}$.

consideration, where local moments sum to zero, which is the resulted global null moment. Therefore, stable FM state is the sole contributor of $\mathrm{T}_{\mathrm{C}}$, whilst making transition from orderedness to disorderedness of spins.

The Figs. 1(a, b) show the energy difference $\Delta \mathrm{E}$ between FM and DLM states versus Ti, Mn, and $\mathrm{Ni}$ concentrations in the host $\mathrm{AlAs}$ and AlP compounds due to ASA calculation, respectively. The graphical patterns are plotted for dopant concentrations of $5 \%$ with dot-dashed line, $10 \%$ with short dashed line, 15\% with dashed line, and 20\% with solid line. Quite surprisingly, zer $\$ \mathrm{E}$ results for $20 \%$
The magnetic properties for a particular $10 \%$ concentration of dopants in AlAs and AlP are shown in Table 1 and Table 2, respectively. The $\mathrm{T}_{\mathrm{C}}$ values of the compounds along with net and spin magnetic moments are tabulated. The bracketed values are calculated using the MT potential approximation, whereas the rest of the data are generated by ASA calculation. In the tables, zerd $\mathrm{E}$ is meant a non-magnetic situation, where FM and DLM sates are energetically equal. In contrast, negature denotes the higher energy situation of FM states and therefore it's not a true FM ground state. In such cases, calculation of $T_{C}$ is absurd, which is 
Table 1. Net magnetic moment per cell and local spin moment per atom in the FM state of $\left(A l_{1-x} T_{x}\right) A s$ and the corresponding energy difference between the FM and DLM states for different TM atoms. The bracketed values shown in the columns $\left(2^{\text {nd }}\right.$ to $\left.5^{\text {th }}\right)$ are calculated using the MT potential approximation for the same concentration.

\begin{tabular}{ccccc}
\hline $\begin{array}{c}\text { Materials } \\
\left(\mathbf{A l}_{\mathbf{1 - \mathbf { x }}} \mathbf{T}_{\mathbf{x}}\right) \mathbf{M}\end{array}$ & $\begin{array}{c}\text { Net moment } \\
\left(\boldsymbol{\mu}_{\mathrm{B}} / \text { cell }\right)\end{array}$ & $\begin{array}{c}\text { Spin moment } \\
\left(\boldsymbol{\mu}_{\mathrm{B}} / \mathbf{a t o m}\right)\end{array}$ & $\Delta \mathbf{E}(\mathbf{m R y})$ & $\mathbf{T}_{\mathbf{C}}(\mathbf{K})$ \\
\hline$\left(\mathrm{Al}_{0.90} \mathrm{Ti}_{0.10}\right) \mathrm{As}$ & $0.099(0.010)$ & $0.769(0.703)$ & $0.134(0.159)$ & $141(167)$ \\
$\left(\mathrm{Al}_{0 \cdot 90} \mathrm{Mn}_{0 \cdot 10}\right) \mathrm{As}$ & $0(0.400)$ & $0(3.730)$ & $0(0.568)$ & $-(597)$ \\
$\left(\mathrm{Al}_{0 \cdot 90} \mathrm{Ni}_{0 \cdot 10}\right) \mathrm{As}$ & $0(0)$ & $0(0)$ & $0(0)$ & $-(-)$ \\
\hline
\end{tabular}

denoted by dashed (-) symbol. Calculated $\mathrm{T}_{\mathrm{C}}$ in Kelvin against concentrations of $\mathrm{Ti}$ and $\mathrm{Mn}$ in AlAs and AlP are shown in Figs. 2(a, b), respectively. The dotted horizontal line is the RT level. Calculated $\mathrm{T}_{\mathrm{C}}$ increases rapidly to a certain value and keep saturated for a certain concentration range. A decreasing trend of $\mathrm{T}_{\mathrm{C}}$ is also clear from the figure above $16 \%$ concentration of the dopant. In Fig. 2(b), it is seen that above 3\% doping concentrations all the
$\left(\mathrm{Al}_{0.90} \mathrm{Ni}_{0.10}\right)$ As is shown, which is a $\mathrm{FM}$ calculation, where spin states are found to have equal density in both spin directions. Thus, total and partial DOS exactly cancel each other and results a zero spin polarization at the Fermi level. In this case both net moment per cell and spin moment per $\mathrm{Ti}$ atom are individually zero. This is the non-magentic behavior of the $\mathrm{Ni}$ doped compound at $10 \%$ doping concentration. However, it is found that the situation alters, that

Table 2. Net magnetic moment per cell and local spin moment per atom in the $F M$ state of $\left(\mathrm{Al}_{1-\mathrm{x}} \mathrm{T}_{\mathrm{x}}\right) \mathrm{P}$ and the corresponding energy difference between the FM and DLM states for different TM atoms. The bracketed values shown in the columns $\left(2^{\text {nd }}\right.$ to $\left.5^{\text {th }}\right)$ are calculated using the MT potential approximation for the same concentration.

\begin{tabular}{ccccc}
\hline $\begin{array}{c}\text { Materials } \\
\left(\mathbf{A l}_{\mathbf{1 - x}} \mathbf{T}_{\mathbf{x}}\right) \mathbf{P}\end{array}$ & $\begin{array}{c}\text { Net moment } \\
\left(\boldsymbol{\mu}_{\mathrm{B}} / \text { cell) }\right.\end{array}$ & $\begin{array}{c}\text { Spin moment } \\
\left(\boldsymbol{\mu}_{\mathrm{B}} / \text { atom) }\right.\end{array}$ & $\Delta \mathbf{E}(\mathbf{m R y})$ & $\mathbf{T}_{\mathbf{C}}(\mathbf{K})$ \\
\hline$\left(\mathrm{Al}_{0.90} \mathrm{Ti}_{0.10}\right) \mathrm{P}$ & $0(0.098)$ & $0(0.617)$ & $0(0.112)$ & $-(118)$ \\
$\left(\mathrm{Al}_{0 \cdot 90} \mathrm{Mn}_{0 \cdot 10}\right) \mathrm{P}$ & $0.40(0)$ & $4.147(0)$ & $0.673(0)$ & $708(-)$ \\
$\left(\mathrm{Al}_{0.90} \mathrm{Ni}_{0 \cdot 10}\right) \mathrm{P}$ & $0.28(0)$ & $2.504(0)$ & $-0.950(0)$ & $-(-)$ \\
\hline
\end{tabular}

calculated $\mathrm{T}_{\mathrm{C}}$ are found to be higher than $\mathrm{RT}$, whereas $\left(\mathrm{Al}_{1-\mathrm{x}} \mathrm{Ti}_{\mathrm{x}}\right)$ As exhibit low $\mathrm{T}_{\mathrm{C}}$ well below RT, as shown in Fig. 2(a).

The investigation of electronic band structures is very important for better understanding of the electronic properties of host aluminium pnictides and their doped alloys. In Fig. 3(a) density of states (DOS) of $\mathrm{Ni}$ doped compound is, FM state is found for excess amount of doping, mainly, more than $90 \%$ doping changes the non-magnetic state to FM state with evolving magnetic moment. The Fig. 3(b) exhibits a DOS of DLM state calculation of $\mathrm{Mn}$ doped $\left(\mathrm{Al}_{0.90} \mathrm{Mn}_{0.05}^{\mathrm{u}} \mathrm{Mn}_{0.05}^{\mathrm{d}}\right) \mathrm{P}$, where ' $\mathrm{u}$ ' and 'd' denote the up spin and down spin configurations. The partial DOS of dotted lines (up spin) and dashed lines (down spin) again cancels them in both 

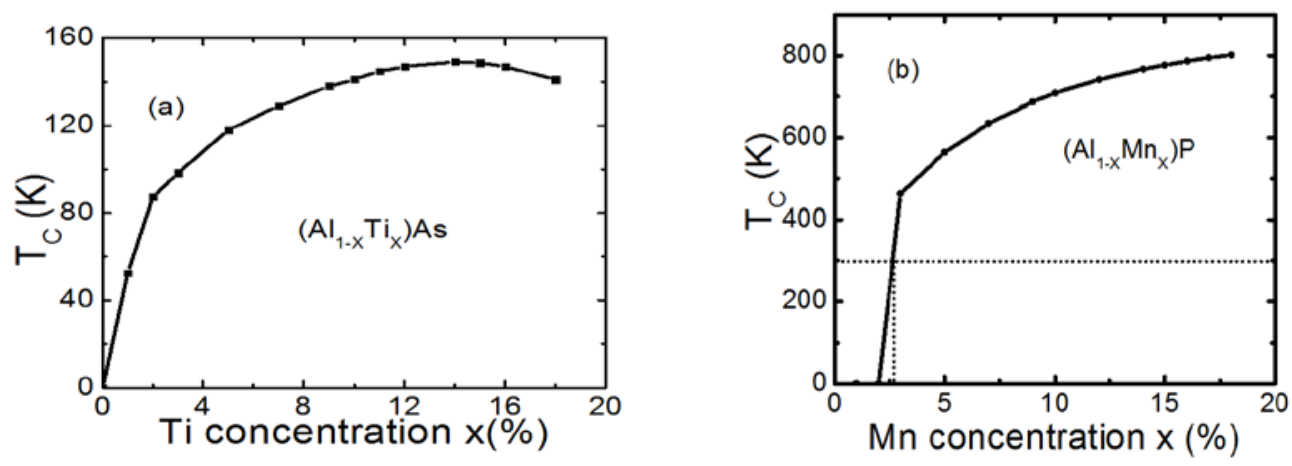

Fig. 2. Curie temperature $T_{C}$ in Kelvin against doping concentrations of (a) $\left(\mathrm{Al}_{1-\mathrm{x}} \mathrm{Ti}_{\mathrm{x}}\right) \mathrm{As}$, and (b) $\left(\mathrm{Al}_{1-\mathrm{x}} \mathrm{Mn}_{\mathrm{x}}\right) \mathrm{P}$ in a range of impurity concentrations $(\mathrm{x} \approx 20 \%)$.

spin directions. Therefore, a null net moment is obtained, where local moments are non-zeros having same magnitude. This DLM state can be simulated as a paramagnetic state calculation of magnetic materials.

The induced magnetic properties calculated by considering ASA in the DMS system can be spin direction with full spin polarization along the up spin direction. The vertical axis represents spin-up and down DOS with a zero level separator dashed line, while the horizontal axis denotes the energy relative to Fermi energy in Rydberg unit. The vertical dashed line at zero energy axis indicates the Fermi level. The
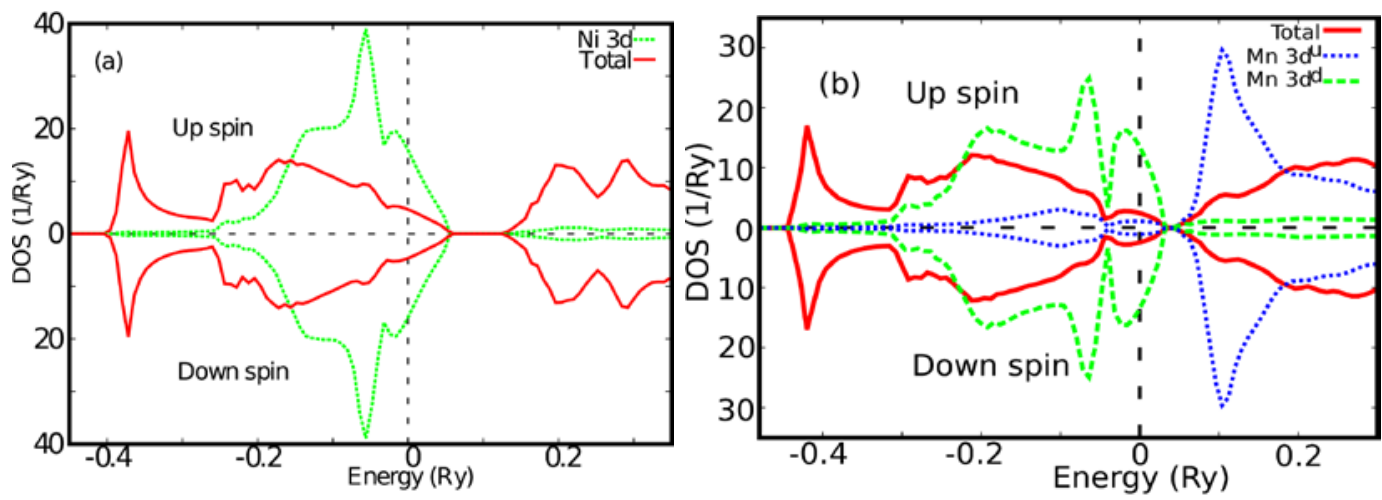

Fig. 3. Total and partial DOS of (a) $\left(\mathrm{Al}_{0.90} \mathrm{Ni}_{0.10}\right)$ As magnetic semiconductor with $\mathrm{Ni}$ 3d states, (b) $\left(\mathrm{Al}_{0.90} \mathrm{Mn}^{\mathrm{u}}{ }_{0.05} \mathrm{Mn}^{\mathrm{d}}{ }_{0.05}\right) \mathrm{P}$ disordered local moment arrangement with $\mathrm{Mn}$ 3d states. The vertical dashed line indicates the Fermi level.

explained by electronic structures. The total and local DOS of $\mathrm{Ti}$ and $\mathrm{Mn}$ doped aluminium pnictides are plotted in Figs. 4(a, b), respectively. The total DOS are evaluated per unit cell and partial DOS are calculated per atomic orbitals. In the DOS pattern, there exists a gap region near the Fermi zone along the down narrow and sharp peaks indicate the localized nature of the $3 d$ electrons. The states are split due to the effect of crystal field splitting, where the electronic states below the Fermi level are fully occupied. The DOS curves confirm that due to the $\mathrm{Ti}$ and $\mathrm{Mn}$ substitution at the $\mathrm{Al}$ site, the compounds have half-metallic character, 

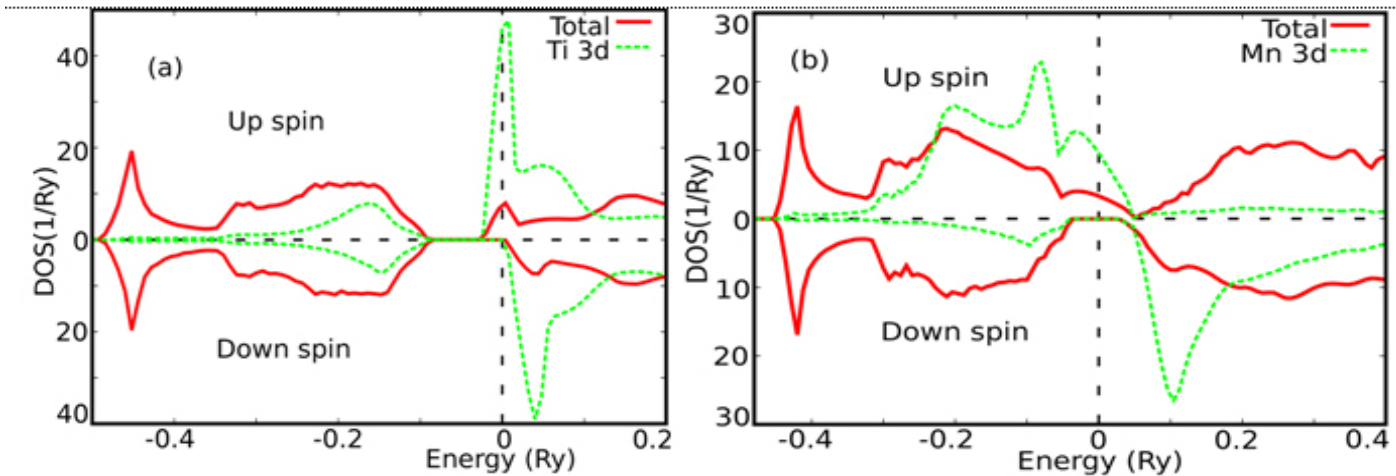

Fig. 4. Total and local $3 d$ DOS of (a) Ti doped $\left(\mathrm{Al}_{0.90} \mathrm{Ti}_{0.10}\right) \mathrm{As}$, and (b) $\mathrm{Mn}$ doped $\left(\mathrm{Al}_{0.90} \mathrm{Mn}_{0.10}\right) \mathrm{P}$ dilute magnetic semiconductors.

because near the Fermi level, majority spins are metallic and minority spins are semiconducting characters.

\section{CONCLUSION}

The KKR-Green's function calculations of multiple scattering theory are performed to understand the role of $\mathrm{Ti}, \mathrm{Mn}$, and $\mathrm{Ni}$ dopants on the magnetic properties and electronic structures of doped aluminium pnictides. Since the calculations are performed assuming spherical approximations to the cell potential, thus for MT potential calculations, charge discrepancy is treated by assigning it to the interstitial region, whereas for ASA calculations, no such option exists. It is found that $\mathrm{Ti}$ doped AlAs and $\mathrm{Mn}$ doped AlP are probable candidates for low and high $\mathrm{T}_{C}$ FM materials, respectively. The $\mathrm{T}_{C}$ higher than RT is found in Mn doped AlP compounds. Such type of half metallic DMS has applications in the field of spintronics, where the dominated magnetism is controlled by electron spins.

\section{ACKNOWLEDGEMENT}

The authors gratefully acknowledge the laboratory support from CARS (Centre for Advanced Research in Sciences), University of Dhaka, for performing the numerical calculations.

\section{REFERENCES}

Akai, H. 2002. http://kkr.issp.u-tokyo.ac.jp.

Anderson, P. W. and H. Hasegawa. 1955. Phys. Rev. 100: $675-681$.

Dietl, T., H. Ohno, F. Matsukura, J. Civert and D. Ferrand. 2000. Science. 287: 1019-1022.

Goodenough, J. B. 1955. Phys. Rev. 100: 564-673.

Kanamori, J. and K. Terakura. 2001. J. Phys. Soc. Jpn. 70: $1433-1434$

Kohn, W. and L. J. Sham. 1965. Phys. Rev. 140: A1133A1138.

Lalngaihawmi, R., B. Vanlalruata, A. Shankar, D. P. Rai, Sandeep and R.K. Thapa. 2014. AIP Conf. Proc. 1661: 050006-1-0560006-11.

Moore, G. E. 1965. Electronics 38: 114-117.

Picket, W. E., S. C. Erwin, O. Mashima, W. R. Lambrecht and B. Segall. 1989. MRS Symp. Proc. No 162.

Sato, K., P. H. Dederics and H. K. Yoshida. 2003 Europhys. Lett. 61: 403-408.

Shahjahan, M. and T. Oguchi. 2016. J. Phys. Chem. Solids 93: 157-162.

Shahjahan, M. and M. J. Sadique. 2018. Computation. Condens. Matter 14: 89-93.

Shiba, H. 1971. Prog. Theor. Phys. 46: 77-94.

Wolf, S. A., D. D. Awschalom, R. A. Buhrman, J. M. Daughton, S. V. Molnár, M. L. Roukes, A. Y Chtchelkanova and D. M. Treger. 2001. Science 294: 1488-1495.

Wyckoff, R. W. G. 1963. Crystal structures, Interscience publishers, $2^{\text {nd }}$ ed, New York.

Zener, C. 1951. Phys. Rev. 82: 403-407.

(Received revised manuscript on 10 July 2018) 